\title{
Dynamic Response Analysis of Medium-Speed Maglev Train with Track Random Irregularities
}

\author{
Peichang Yu $(\mathbb{D}$, Mengxiao Song $\mathbb{D}$, Qiang Chen, Lianchun Wang, Guang He $\mathbb{D}$, \\ and Peng Leng \\ College of Intelligence Science and Technology, National University of Defense Technology, Changsha 410073, China \\ Correspondence should be addressed to Mengxiao Song; songmengxiao14@nudt.edu.cn
}

Received 12 April 2021; Revised 13 August 2021; Accepted 22 September 2021; Published 5 November 2021

Academic Editor: Dung-Ying Lin

Copyright ( $) 2021$ Peichang Yu et al. This is an open access article distributed under the Creative Commons Attribution License, which permits unrestricted use, distribution, and reproduction in any medium, provided the original work is properly cited.

\begin{abstract}
In order to analyze the dynamic response of medium-speed maglev train in the speed range of $0-200 \mathrm{~km} / \mathrm{h}$, the suspension performance, suspended energy consumption, and riding comfort of the train stimulated by random track irregularities are discussed in this paper. Firstly, the model of medium-speed maglev train including car body, air spring vibration isolation system, and the suspension system is established. Then, a controller based on flux inner feedback loop and PID outer feedback loop is designed for the suspension system. The established model is stimulated by the actual track power spectrum in full speed range. The simulation results show that the fluctuation of suspension gap is less than $\pm 4 \mathrm{~mm}$. Furthermore, thanks to the adding of permanent magnet, the power consumption is significantly reduced, which is of benefit to the electromagnet heating problem and on-board levitation power supply system. The riding comfort of the train moving on the irregular track using Sperling index is assessed. The experimental results validate the effectiveness of the proposed analytical calculation model of medium-speed maglev train. It is shown that medium-speed maglev train achieved good performance, significant power reduction, and satisfactory riding comfort.
\end{abstract}

\section{Introduction}

As a new type of rail transportation, maglev train applies electromagnetic force to support the vehicle without contact and is propelled by linear motor. Compared with the traditional wheel-rail train, maglev train avoids the mechanical contact between the vehicle and the track, which has the advantages of low noise, small turning radius, and low maintenance cost. Researchers in Germany, Japan, China, South Korea, Brazil, and other countries have studied kinds of maglev train technology [1-5].

In recent years, low-speed maglev trains have developed rapidly in China, South Korea, and Japan. Low-speed maglev train is mainly used for urban traffic. In 2016, the Changsha Airport Maglev Express line (Figure 1(a)) began to operate, with a total length of 18.2 kilometers [2]. In 2011, Beijing started construction of the S1 line (Figure 1(b)), which is the first low-speed maglev commercial demonstration line in China. South Korea has built a low-speed maglev transportation line at Incheon Airport (Figure 1(c)), which began to operate in February 2016 [6]. In Japan, the low- and medium-speed maglev system using HSST technology has been officially put into commercial operation in Aichi Prefecture in March 2005 (Figure 1(d)) [3]. In addition, researchers in the United Kingdom, the United States, Italy, Russia, and other countries have also made contributions to maglev trains $[7,8]$. It is clearly shown that the technologies of low-speed maglev train are mature.

In recent years, in order to offer an alternative method of transportation between large cities and satellite cities, China has studied the medium-speed hybrid levitation maglev train with the highest speed of $200 \mathrm{~km} / \mathrm{h}$. In 2018, the medium-speed maglev train with the highest speed of $160 \mathrm{~km} / \mathrm{h}$ (Figure 2) was developed by the National University of Defense Technology [9]. This type of maglev train applies both the electromagnet and the permanent magnet as a hybrid suspension structure, which can effectively reduce suspension power consumption and improve suspension 


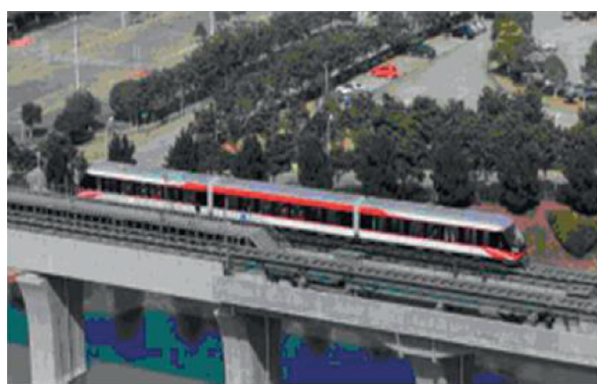

(a)

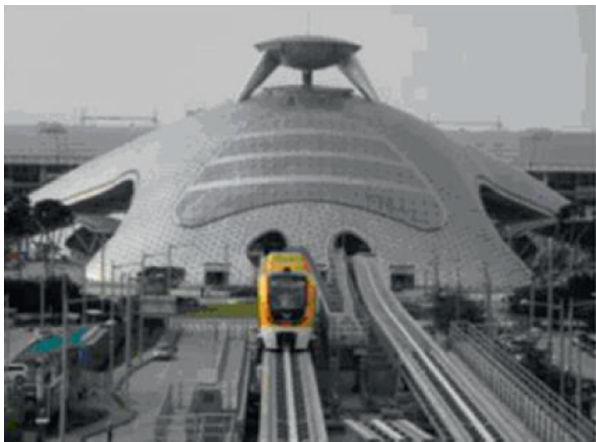

(c)

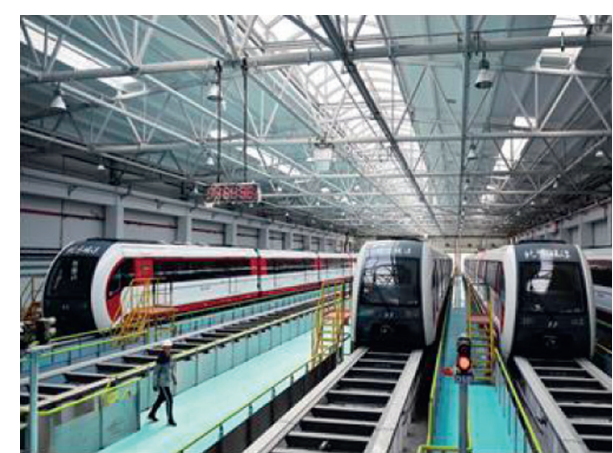

(b)

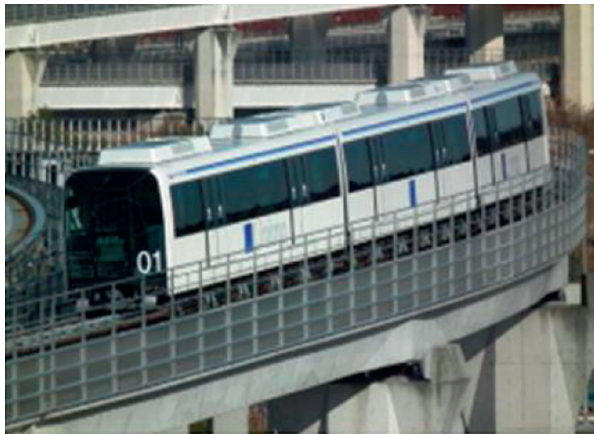

(d)

FIGURE 1: Operating medium- and low-speed maglev lines. (a) Changsha Airport Maglev Express. (b) Beijing S1 maglev line. (c) Incheon Airport maglev line. (d) Japan HSST-100L low-speed maglev train.

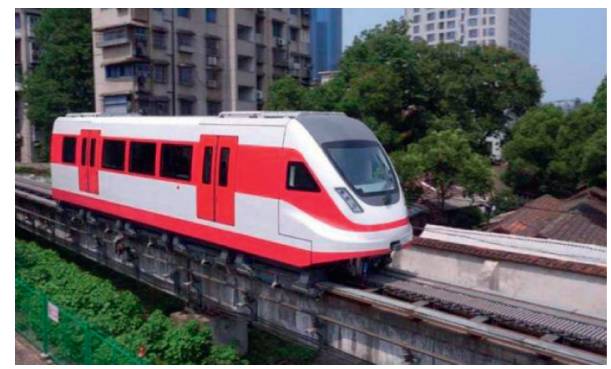

Figure 2: $160 \mathrm{~km} / \mathrm{h}$ type medium-speed maglev train.

efficiency. At the same time, linear synchronous motor can further increase the running speed to $200 \mathrm{~km} / \mathrm{h}$ effectively. However, there are still some problems of medium-speed maglev train as follows that have not been intensively studied.

Firstly, the levitation control technology for mediumspeed $(0-200 \mathrm{~km} / \mathrm{h})$ maglev train has not been studied before. Many scholars have conducted researches on this issue, and many control strategies have been discussed, such as backstepping controller [10], PID controller based on cascade idea [11], and model reference adaptation [12]. However, these researches are mainly based on the static or the low-speed situation. It is not clear if these control strategies can be applied under the circumstance of $200 \mathrm{~km} / \mathrm{h}$.

Secondly, the suspension power consumption of the whole vehicle with different loads and at different speeds has not been studied before. In order to reduce the power consumption of the levitation system, permanent-electromagnetic hybrid structure attracts much attention. Papers $[13,14]$ analyzed the design of suspension electromagnets and the optimization of permanent magnets and made static tests. They have pointed out that the hybrid suspension system has good energy-saving effects under static conditions. However, suspension power consumption at different operating speeds is not clear. In order to design and optimize the suspension power supply system for medium-speed maglev trains, it is necessary to analyze the suspension power consumption of the whole vehicle with different loads and at different speeds.

At last, the riding comfort in the range of $0-200 \mathrm{~km} / \mathrm{h}$ has not been considered before. As a passenger transport, the riding comfort of maglev trains is the focus. Aiming at this problem, Zhao and others [15, 16] studied the comfort of high-speed maglev trains with the Sperling index. He et al. 
[17] also analyzed the medium-speed maglev, but the riding comfort in the speed range of $0-200 \mathrm{~km} / \mathrm{h}$ is not considered.

In this paper, an analytical calculation model of whole maglev train including vehicle body, secondary system, and suspension module is established in Section 2. In Section 3, according to the characteristics of hybrid suspension, a suspension controller based on the magnetic flux feedback inner loop and the gap feedback outer loop is designed. In Section 4, a comprehensive simulation analysis of mediumspeed maglev trains with different loads and at different speeds is carried out with the stimulation of actual track, focusing on the suspension performance, suspension power consumption, and the riding comfort. In Section 5, experiments are made to validate the analytical model and the simulation. Section 6 concludes this study.

\section{Dynamic Modeling of Medium-Speed Maglev Train}

A section of a medium-speed maglev train is shown in Figure 3. The body structure of the medium-speed maglev train is basically the same as that of the low-speed maglev train. The main difference lies in the levitation and linear traction mode. The maglev train can be divided into three parts.

Vehicle body: it is the upper body structure for passengers. The load changes greatly and the maximum design load is 12 tons. Riding comfort is the core problem. Therefore, in practice, the vehicle dynamics response is reflected by the vertical acceleration along the $z$-axis and the two angular accelerations around the $x$-axis and $y$-axis of the vehicle body.

Air spring: it is the intermediate vibration isolation structure, which connects the car body and the lower suspension control system. Its cut-off frequency is generally $2 \mathrm{~Hz}$, which can effectively isolate high-frequency vibration and improve the riding comfort. As a basis for system modeling, the air spring can be equivalent to a springdamping system.

Levitation module: it is the core of the vehicle suspension. A single levitation control system includes levitation sensors, hybrid levitation electromagnets, and levitation controllers.
2.1. Modeling of Vehicle Body. In the analytical calculation model, this paper mainly studies the vibration, pitch, and roll characteristics of the vehicle. Because the main consideration in this article is the impact of the suspension system on the car body, the source of force is mainly transmitted through the air spring, and the established model is shown in Figure 4 . The related parameters are defined as follows:

$F_{s z i j}$ : vertical force of the $j$-th air spring on the $i$-th suspension module of the vehicle body

$m_{c} g$ : gravity of vehicle body

$a_{c}$ : vertical acceleration of vehicle body

$\phi_{c}$ : angular acceleration of the car body around the $x$-axis

$\theta_{c}$ : angular acceleration of the car body around the $y$-axis

$C_{i}$ : distance between air springs and the center of the car body in the $x$-direction

$w_{c}$ : distance between air springs and the center of the car body in the $y$-direction

Icy: moment of inertia of the car body around the $y$-axis Icx: moment of inertia of the car body around the $x$-axis

During operation, the vertical force of the vehicle includes two aspects. One is its own gravity $m_{c} g$, and the other is the levitation force $F_{s z i j}$ transmitted by the levitation electromagnet through 20 air springs. In order to study the state of the vehicle body during operation, the focus is on the vehicle's vertical vibration acceleration $a_{c}$, the vehicle's rotational angular acceleration around the $x$-axis direction, and the angular acceleration around the $y$-axis direction.

The force of the car body is shown in Figure 4, and the relationship of the car body's vertical movement, pitching, and overturning can be calculated.

Vehicle body vertical motion is expressed in the following equation:

$$
m_{c} \ddot{z}_{c}=m_{c} g-\sum_{i=1}^{10} \sum_{j=1}^{2} F_{s z i j} .
$$

Vehicle body pitch motion is expressed in the following equation:

$$
\begin{aligned}
I_{c y} \ddot{\theta}_{c}= & \left(F_{s z 51}+F_{s z 61}-F_{s z 52}-F_{s z 62}\right) c_{1} \cos \theta_{c}+\left(F_{s z 32}+F_{s z 42}-F_{s z 71}-F_{s z 81}\right) c_{2} \cos \theta_{c} \\
& +\left(F_{s z 31}+F_{s z 41}-F_{s z 72}-F_{s z 82}\right) c_{3} \cos \theta_{c}+\left(F_{s z 12}+F_{s z 22}-F_{s z 91}-F_{s z 101}\right) c_{4} \cos \theta_{c} \\
& +\left(F_{s z 11}+F_{s z 21}-F_{s z 92}-F_{s z 102}\right) c_{5} \cos \theta_{c} .
\end{aligned}
$$

Vehicle body roll motion is expressed in the following equation:

$$
I_{c x} \ddot{\phi}_{c}=w_{c}\left(\sum_{i=2,4,6,8,10} \sum_{j=1}^{2} F_{s z i j}-\sum_{i=1,3,5,7,9} \sum_{j=1}^{2} F_{s z i j}\right) .
$$

The above three equations can describe the car body motion with three degrees; this is the basis for the analysis of the train.

2.2. Modeling of Air Spring. Air spring, thanks to the good vibration isolation character, is currently used in maglev 


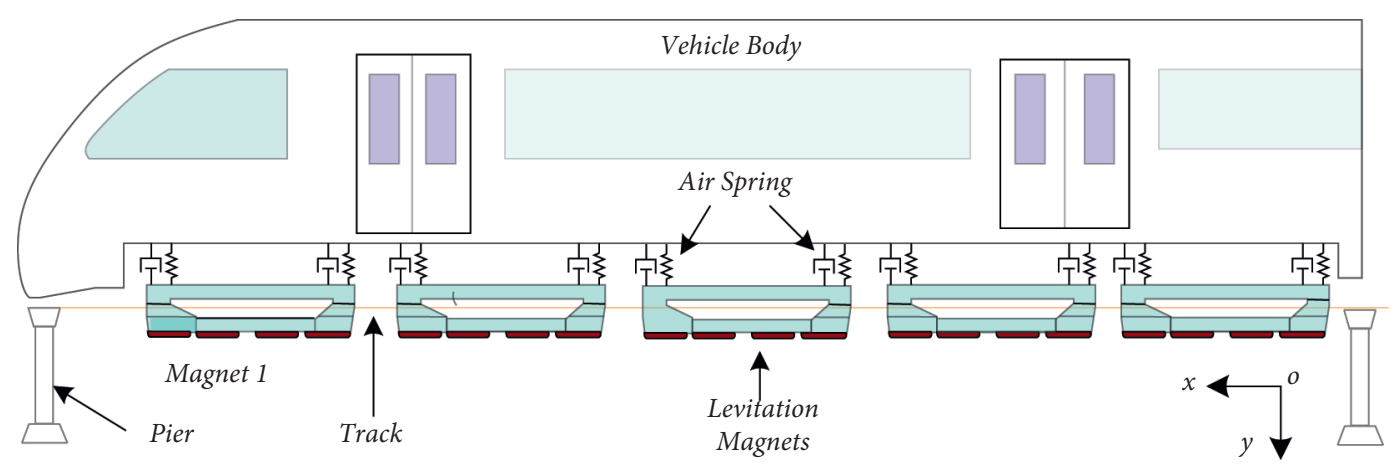

Figure 3: Side view of medium-speed maglev train.

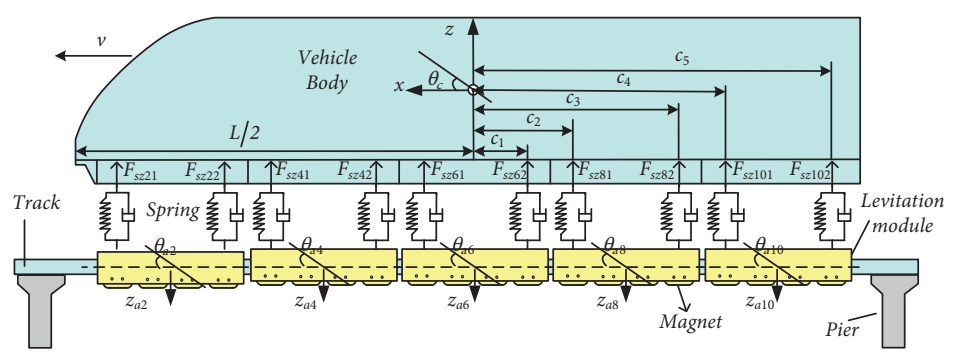

Side view

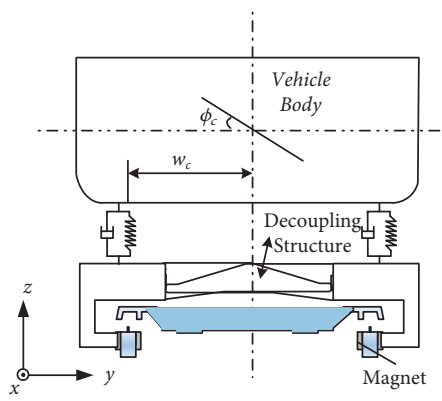

Sectional view

Figure 4: Schematic diagram of vehicle body.

trains for isolating vibration between suspension modules and the vehicle body. The modeling of air spring involves the category of fluid mechanics, and it is very difficult to describe it accurately by mathematical means. This study conducted a frequency domain analysis on the stiffness and damping characteristics of air springs used in low- and medium-speed maglev trains through experimental test. The air spring can be simplified into the mechanical model shown in Figure 5.

When the maglev train is statically suspended on the track, assuming that the gravity of the car body causes the initial deformation of the air spring to be $z_{c 0}$, each maglev train has 20 suspension points; then

$$
F_{s z i j}=z_{c 0} k_{s}=\frac{m_{c} g}{20} \text {. }
$$

When the maglev train is running dynamically, from the mechanical model of the air spring shown in Figure 5, the calculation equation for the secondary suspension force provided by the 20 air springs of the vehicle can be obtained as

$$
\begin{aligned}
F_{s z i j}= & z_{c 0} k_{s}+k_{s}\left(z_{c}-c_{i} \theta_{c}-z_{a i}+l_{b m i} \theta_{a i}\right) \\
& +c_{s}\left(\dot{z}_{c}-c_{i} \dot{\theta}_{c}-\dot{z}_{a i}+l_{b m i} \dot{\theta}_{a i}\right)
\end{aligned}
$$

where $z_{a i}$ is the vertical displacement of the $i$-th electromagnet, $l_{b m i}$ is the moment of inertia of the $i$-th levitation electromagnet, and $\theta_{a i}$ is the pitch angle of the $i$-th electromagnet. The above equation shows that the secondary suspension force provided by the air spring is related to the compression amount of the air spring and its speed. The compression amount and speed of the air spring can be determined by the suspension gap and pitch angle of the suspension module and pitch angle, vertical displacement, and rolling angle of the vehicle body.

2.3. Dynamic Modeling of the Suspension Module. The maglev train has 10 independent levitation modules. The levitation electromagnets are numbered from 1 to 10 , and the electromagnets on the left track (as shown in Figure 6) are defined as $2,4,6,8$, and 10 .

The force schematic diagram of the suspended module is shown in Figure 6. Regardless of the linear motor's traction on the suspension module, the forces received by the suspension module include gravity, electromagnetic force (including vertical suspension force and lateral guiding force), and the vertical effect of the car body on the suspension module through the air spring force. A single suspension module contains four electromagnet wire packages, which are generally divided into 2 suspension points for control.

The symbols in Figure 6 are explained as follows:

$F_{m i k}$ : the levitation force generated by the $k$-th magnet wire packages of the $i$-th levitation module

$F_{\text {smij: }}$ : the vertical pressure of the $j$-th air spring on the $i$-th suspension module on the suspension module

$m_{a}$ : levitation module mass

$Z_{a i}$ : vertical movement distance of the $i$-th suspension module

(1) Vertical movement of levitation module is 


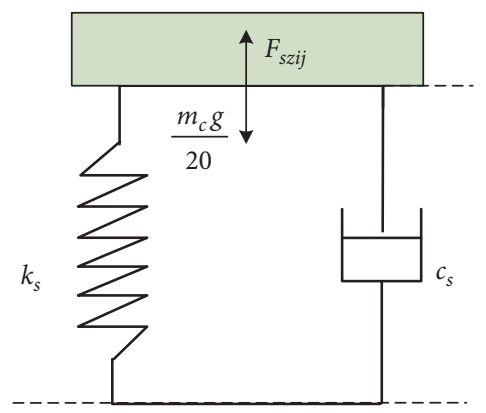

Figure 5: Simplified mechanical model of air spring under static conditions.

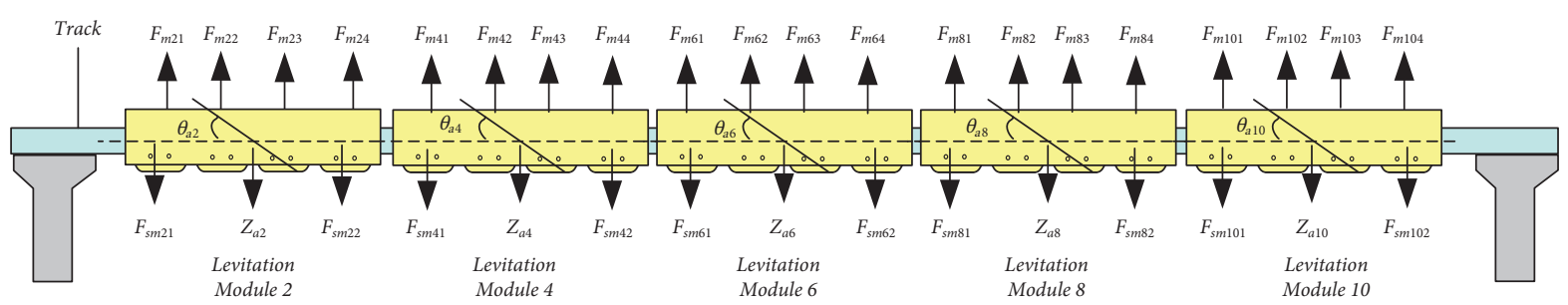

FIGURE 6: Schematic diagram of the force on levitation modules.

$$
m_{a i} \ddot{z}_{a i}=\sum_{j=1}^{2} F_{s m i j}-\sum_{k=1}^{4} F_{m i k}+m_{a i} g .
$$

(2) Pitch motion of levitation module is

$$
\begin{aligned}
I_{c y i} \ddot{\theta}_{a i}= & l_{b m}\left(F_{s z i 1}-F_{s z i 2}\right) \cos \theta_{a i} \\
& +\left(\sum_{k=1}^{2} F_{m i k} l_{m k}-\sum_{k=3}^{N} F_{m i k} l_{m k}\right) \cos \theta_{a i} .
\end{aligned}
$$

The movement of the suspended electromagnet is in two directions: one is its flip angle, and the other is vertical vibration. So, the movement relationship of the suspended electromagnet module can be described.

Based on the analysis above, we can establish the dynamic model of the maglev train, which includes the threedegree-of-freedom model of the car body, the secondary equivalent model based on the hollow spring, and the motion equation of the suspension module. It can be summarized as follows:

$$
\left\{\begin{array}{l}
\ddot{\phi}_{c}=\frac{w_{c}}{I_{c x}}\left(\sum_{i=2,4,6,8,10} \sum_{j=1}^{2} F_{s z i j}-\sum_{i=1,3,5,7,9} \sum_{j=1}^{2} F_{s z i j}\right), \\
\ddot{z}_{c}=g-\sum_{i=1}^{10} \sum_{j=1}^{2} \frac{F_{s z i j}}{m_{c}}, \\
\ddot{\theta}_{c}=\frac{1}{I_{c y}}\left[\left(F_{s z 51}+F_{s z 61}-F_{s z 52}-F_{s z 62}\right) c_{1} \cos \theta_{c}+\left(F_{s z 32}+F_{s z 42}-F_{s z 71}-F_{s z 81}\right) c_{2} \cos \theta_{c}\right. \\
\left.+\left(F_{s z 31}+F_{s z 41}-F_{s z 72}-F_{s z 82}\right) c_{3} \cos \theta_{c}+\left(F_{s z 12}+F_{s z 22}-F_{s z 91}-F_{s z 101}\right) c_{4} \cos \theta_{c}+\left(F_{s z 11}+F_{s z 21}-F_{s z 92}-F_{s z 102}\right) c_{5} \cos \theta_{c}\right], \\
F_{s z i j}=z_{c 0} k_{s}+k_{s}\left(z_{c}-c_{i} \theta_{c}-z_{a i}+l_{b m i} \theta_{a i}\right)+c_{s}\left(\dot{z}_{c}-c_{i} \dot{\theta}_{c}-\dot{z}_{a i}+l_{b m i} \dot{\theta}_{a i}\right), \\
\ddot{z}_{a i}=\frac{1}{m_{a i}} \sum_{j=1}^{2} F_{s m i j}-\frac{1}{m_{a i}} \sum_{k=1}^{4} F_{m i k}+g, \\
\ddot{\theta}_{a i}=\frac{1}{I_{c y i}} l_{b m}\left(F_{s z i 1}-F_{s z i 2}\right) \cos \theta_{a i}+\frac{1}{I_{c y i}}\left(\sum_{k=1}^{2} F_{m i k} l_{m k}-\sum_{k=3}^{N} F_{m i k} l_{m k}\right) \cos \theta_{a i} .
\end{array}\right.
$$


The equation above builds a vehicle dynamics model of the medium-speed maglev train. The levitation system is an unstable system. It is necessary to study the levitation system and design the controller.

\section{Design of Suspension Control System}

A stable, reliable, and excellent levitation controller is the first requirement of the levitation system. Aiming at this system, a suspension control algorithm based on the serial cascade idea is obtained. Magnetic flux density feedback instead of traditional current feedback, combined with mature PID control strategies as the outer loop, can simplify the block diagram structure of levitation control and improve system performance [14, 18, 19].

As shown in Figure 7, the left and right ends of the levitation module are regarded as two levitation control units. Each levitation control unit consists of two seriesconnected solenoid coils with same parameters, sensors (including gap, current, and acceleration sensors), controllers, and other components.

3.1. Modeling of Levitation Module. Model for the suspension module is built in this section. Some assumptions are made. On the one hand, the influence of the vehicle deformation is ignored. The high-frequency vibration of bogie will be isolated by air spring, so the natural mode of the vehicle will not be motivated. On the other hand, considering the small working gap and the allowable engineering error, the magnetic resistance of the iron core and the track will be ignored. The magnetic field will decline down uniformly in the suspended air gap [16]. Irregularity tracksuspension module-car body model is shown in Figure 8. One suspension module is divided into two control points consisting of two electromagnets, where the detecting position and air spring working point acted on both ends of the module.

The suspension module provides magnetic force for the maglev train. $F_{m i j}$ is the magnetic force acted on the $j$-th magnet of the $i$-th suspension module. $\mu_{0}$ is the magnetic permeability. $A$ is the effective area of the magnetic pole. $N$ is the number of coil turns. $i_{i j}$ and $\bar{\delta}_{i j}$ are the current in magnetic coil and the average gap between the magnet and track separately. So, the levitation force of each coil can be written as [20]

$$
\begin{aligned}
F_{m i j}(t) & =\frac{\mu_{0} \mathrm{AN}^{2}}{4}\left(\frac{i_{i j}(t)}{\bar{\delta}_{i j}(t)}\right)^{2}, \\
\bar{\delta}_{i j}(t) & =\frac{1}{2 L_{e}} \int_{x_{m i}+(j-3) L_{e}}^{x_{m i}+(j-2) L_{e}}[\delta(x)] \mathrm{d} x .
\end{aligned}
$$

$x_{m i}$ is the position of the $i$-th module midpoint. $L_{e}$ is the length of the electromagnet coil package. $\delta(x)$ is the gap between the suspension module and track at $x . \delta(x)$ contains two aspects of information: track irregularity and relative position information between the suspended module and the standard track.
3.2. Electrical Model of Levitation Electromagnet. Different from the electromagnetic levitation system, the hybrid levitation system not only includes an electromagnetic coil but also connects a permanent magnet in series with the magnetic field loop. When the system is working at the rated levitation position, the levitation system is mainly provided by permanent magnets and the electromagnet. By the adjustment of the control voltage $u(t)$, electromagnet generates a dynamic force to maintain the stability of the levitation. The advantage of this structure is that it can greatly reduce the energy consumption of the suspension system and even achieve zero current suspension.

The simplified model structure of the hybrid suspension electromagnet is shown in Figure 9, where the deformation of the track and the leakage of the air gap are ignored. The electromagnet is a $U$-shaped structure, containing $N$ turns of electromagnetic coil, controlled by the external power supply voltage $u(t)$, internally generated current $i(t)$, the magnetic flux generated by the electromagnetic coil, and permanent magnet with thickness $h_{m p}$. The suspension gap is $\delta_{e}(t)$. The resulting magnetic flux density is $B(t)$.

For the $j$-th magnet of the $i$-th suspension module of the medium-speed maglev train, the input voltage is $u_{i j}(t) . R$ is the resistance of the coil. $N$ is the number of coil turns. The current voltage equation of the electromagnet coil package can be expressed as [14]

$$
\frac{u_{i j}(t)}{2}=i_{i j}(t) R+\frac{\mu_{0} \mathrm{AN}^{2}}{2 \bar{\delta}_{i j}(t)} \dot{i}_{i j}(t)-\frac{\mu_{0} \mathrm{AN}^{2} i_{i j}(t)}{2 \bar{\delta}_{i j}^{2}(t)} \dot{\bar{\delta}}_{i j}(t) .
$$

The relationship between the voltage in the electromagnetic coil and the magnetic flux density in the hybrid suspension system can be expressed as

$$
u_{i j}(t)=i_{i j}(t) R+\mathrm{NA} \frac{\mathrm{d} B_{i j}}{\mathrm{~d} t},
$$

where flux density $B_{i j}$ can be expressed as

$$
B_{i j}(t)=\frac{\mu_{0}\left(N i_{i j}(t)+H_{c} h_{m p}\right)}{2 z_{i j}(t)+h_{m p} A /\left(\mu_{r} A_{p}\right)} .
$$

Based on equations (12) and (13), it can be seen that the magnetic flux density not only includes the magnetic flux density generated by the electromagnetic coil current but also includes the magnetic flux density generated by the permanent magnet. Moreover, the magnetic flux density of two different sources is not a simple superposition relationship. Current, air gap, height, and permanent magnet thickness are the impact factors of the system. It is a challenge for the controller design.

3.3. Design of PID Controller Based on Magnetic Flux Density Feedback. The hybrid suspension system is an inherently unstable system. The suspension system usually takes the fixed suspension clearance $z_{0}$ as the control target, which requires the fluctuation range of the suspension gap to be within a certain range, so as to ensure that the suspension electromagnet does not contact with the track. 


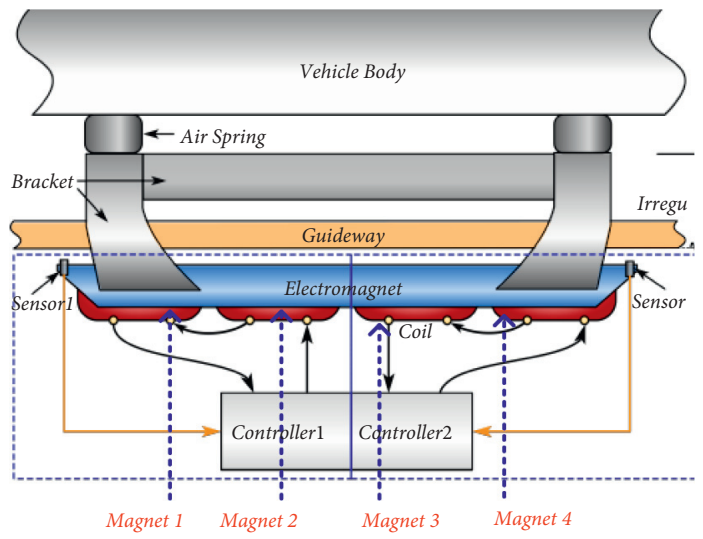

Figure 7: Schematic diagram of the suspension system.

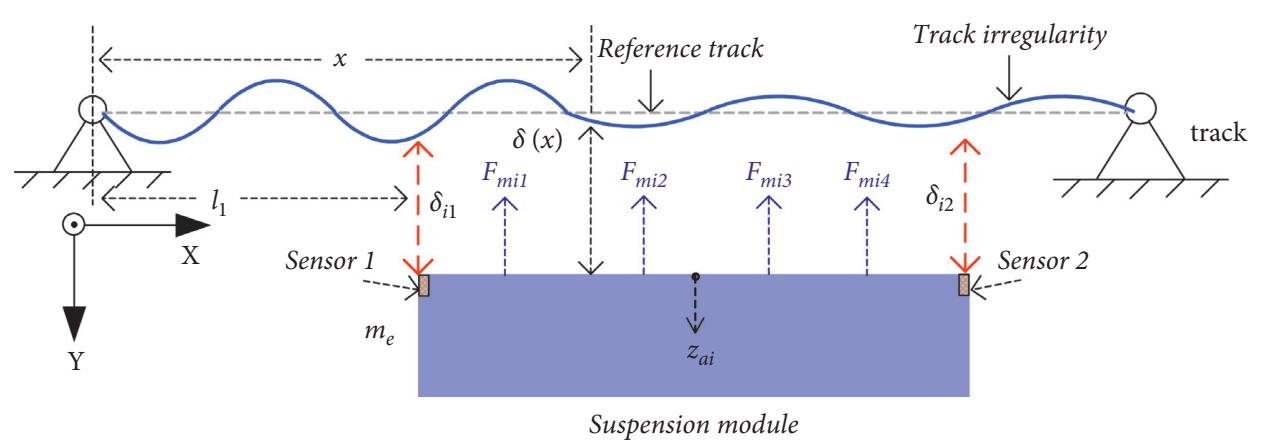

FIgURE 8: Model of separated suspension module.

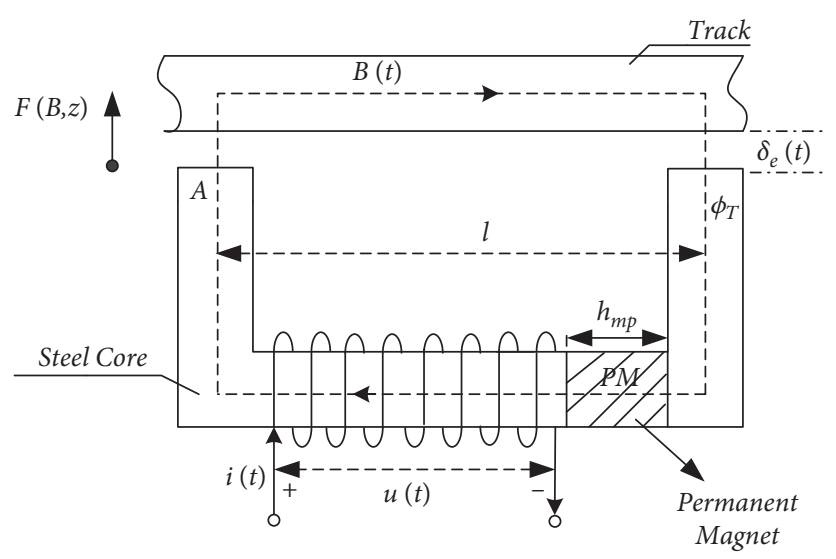

FIGURE 9: Schematic diagram of simplified model of hybrid suspension electromagnet.

Firstly, a controller needs to be designed to ensure its stability. A PID controller, commonly used in industrial control, is chosen as the outer loop of the levitation controller, and the magnetic flux density feedback is chosen as the inner loop of the system. The designed control block diagram is shown in Figure 10.

The expected magnetic flux density is

$$
B_{\exp }(t)=k_{p}\left[z(t)-z_{0}\right]+k_{i} \int_{0}^{t}\left[z(t)-z_{0}\right] \mathrm{d} t+k_{d} \dot{z}(t)+B_{0},
$$

where $k_{p}, k_{i}$, and $k_{d}$ are the parameters of the controller, $z_{0}$ is the rated levitation gap, and $B_{0}$ is the steady-state magnetic flux density value during steady-state levitation, which is used to compensate the gravity of the levitation module.

$$
B_{0}=\sqrt{\frac{\mu_{0} M_{a} g}{A}}
$$

$M_{a}$ is the total mass of the load, including the mass of the module and car body. A magnetic flux density loop is used as the control inner loop, whose purpose is to improve the response speed of the system. In the magnetic flux density 


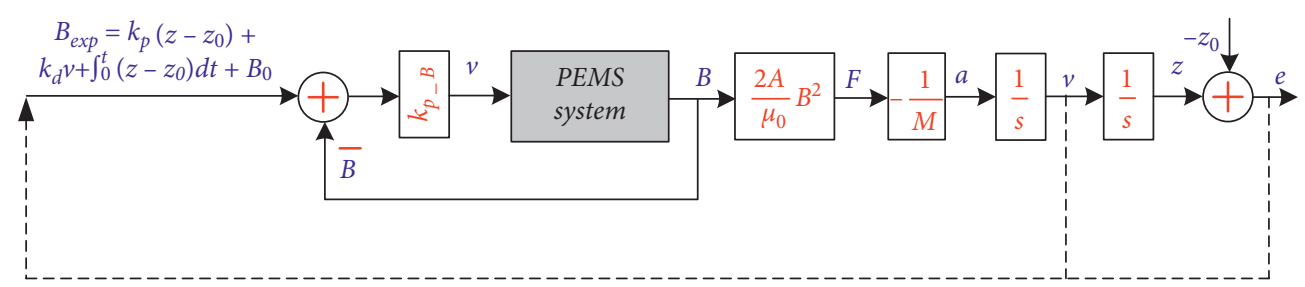

FigURE 10: PID control block diagram of levitation system with flux density feedback.

inner loop, the relationship between the control voltage and the magnetic flux density can be expressed as follows:

$$
u_{c}(t)=k_{p_{-} B}\left[B_{\exp }(t)-B(t)\right] .
$$

There are many ways to obtain $B$ such as observation methods and measurement methods [21].

\section{Numerical Simulation}

Based on the models established in Section 2 and 3, in this section, the vehicle's levitation performance, levitation power consumption, and riding comfort with different loads and at different speeds are analyzed and discussed with the input of actual track irregularity.

4.1. Track Power Spectrum. As shown in equation (17), $S(f)$ is power spectrum of track irregularity. $f(1 / m)$ is the spatial frequency. $A, B, C, D, E, F$, and $G$ are spectral characteristic parameters [22].

$$
s(f)=\frac{A\left(f^{2}+B \cdot f+C\right)}{f^{4}+D \cdot f^{3}+E \cdot f^{2}+F \cdot f+G} .
$$

Referring to the orbit spectrum density function successfully applied in domestic and foreign, Tangshan low-speed maglev track spectrum is chosen to express the orbit spectrum characteristics [9], and the parameters are shown in Table 1.

The track irregularity in Figure 11 is deduced based on formula (17) and Table 1. It will be used as an excitation input to verify the levitation system performance at different speeds in the next step.

4.2. Analysis of Suspension Performance. In order to investigate the performance of the suspension system under the existing track conditions, taking the track irregularity shown in Figure 11 as the excitation, the fluctuation of suspension clearance of suspension module is simulated and analyzed when the vehicle runs at speeds of $40 \mathrm{~km} / \mathrm{h}, 80 \mathrm{~km} / \mathrm{h}, 120 \mathrm{~km} / \mathrm{h}$, $160 \mathrm{~km} / \mathrm{h}$, and $200 \mathrm{~km} / \mathrm{h}$.

In Figure 12, it can be seen that the levitation gap fluctuations of no. 6 levitation modules are maintained within $\pm 4 \mathrm{~mm}$ at different speeds. When the operating speed is below $80 \mathrm{~km} / \mathrm{h}$, the suspension gap fluctuation is within $\pm 3 \mathrm{~mm}$. When the speed is further increased, the fluctuation of the suspension gap becomes larger. The probability of the suspension gap fluctuation within $\pm 4 \mathrm{~mm}$ in full speed range is greater than $99 \%$. This shows that the levitation system can meet the levitation performance requirements under the excitation of the low-speed maglev track irregularity in the speed range of $0-200 \mathrm{~km} / \mathrm{h}$.

In order to further investigate the suspension performance of the vehicle, Figure 13 shows the suspension gap of five suspension modules on one side of the vehicle when the vehicle is running at speed of $200 \mathrm{~km} / \mathrm{h}$ and loaded with $35 \mathrm{t}$. The suspension gap fluctuations of different suspension modules are relatively consistent. The gap fluctuation is less than $\pm 4 \mathrm{~mm}$, and the suspension characteristics are similar, which indicates that different suspension modules are decoupled from each other. The control performance is the same. The same suspension control strategy can meet the requirements of suspension performance. It is also shown that the application of PID levitation control strategy based on magnetic flux feedback can meet the stable and reliable requirements of the levitation system.

4.3. Analysis of Suspension Energy Consumption. For the suspension system, the power consumption is mainly concentrated in the suspension electromagnet coil resistance and the loss of the suspension chopper inside the controller. The loss of the chopper can be reduced by the optimization of the device, so it is not considered here.

Compared with the pure electromagnetic magnet, the suspension current can be greatly reduced due to the addition of the permanent magnet, which can greatly reduce the suspension energy consumption.

Under static conditions, with the same vehicle mass $M_{v g}$, the suspension energy consumption $P$ of the vehicle's ten suspension modules can be expressed as

$$
P=\sum_{i=1}^{10} \sum_{j=1}^{2} P_{i j}
$$

where $P_{i j}$ is the $i$-th levitation module's $j$-th point. As the pure electromagnetic type, the suspension power $P_{i j}$ is

$$
P_{i j}=\frac{4 F_{k} z_{0}^{2}}{A \mu_{0} N^{2}} R
$$

The suspension power $P_{i j}$ of the permanent magnetelectromagnetic hybrid type is

$$
P_{i j}=\left[\frac{\sqrt{\mu_{0} F_{k} / A}\left(2 z_{0}+h_{m p} A /\left(\mu_{r} A_{p}\right)\right)-\mu_{0} H_{c} h_{m p}}{\mu_{0} N}\right]^{2} R .
$$

In the above equation, $F_{k}=M_{v g} / 20$; it is the average load of each suspension point. Under static conditions, the air spring can evenly distribute the mass of the carriage to each suspension module. $z_{0}$ is a stable levitation gap. 
TABLE 1: Characteristic parameters of Tangshan low-speed maglev track spectrum.

\begin{tabular}{lccccccc}
\hline Parameter & $A$ & $B$ & $C$ & $D$ & $E$ & $F$ & $G$ \\
\hline Value & 0.003782 & -0.087513 & 0.001952 & -0.213341 & 0.017071 & -0.000607 & $8.07424 e-6$ \\
\hline
\end{tabular}

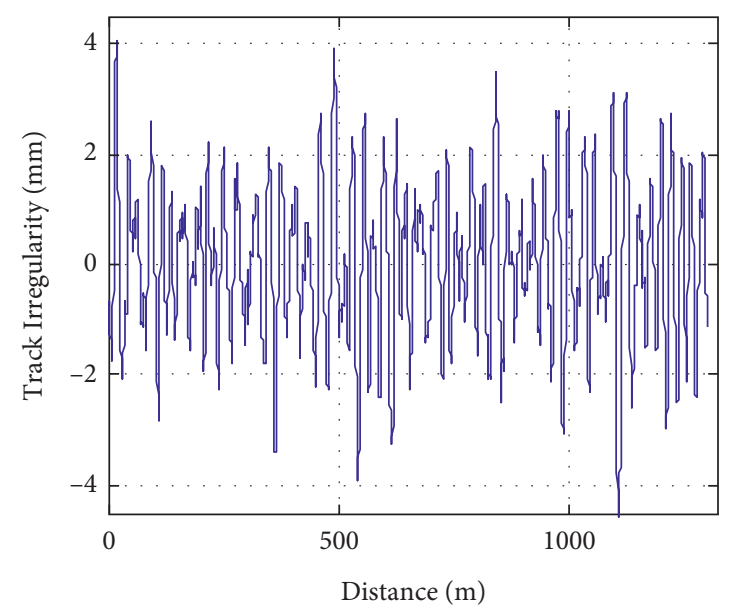

FIgURE 11: Track irregularity with different position.
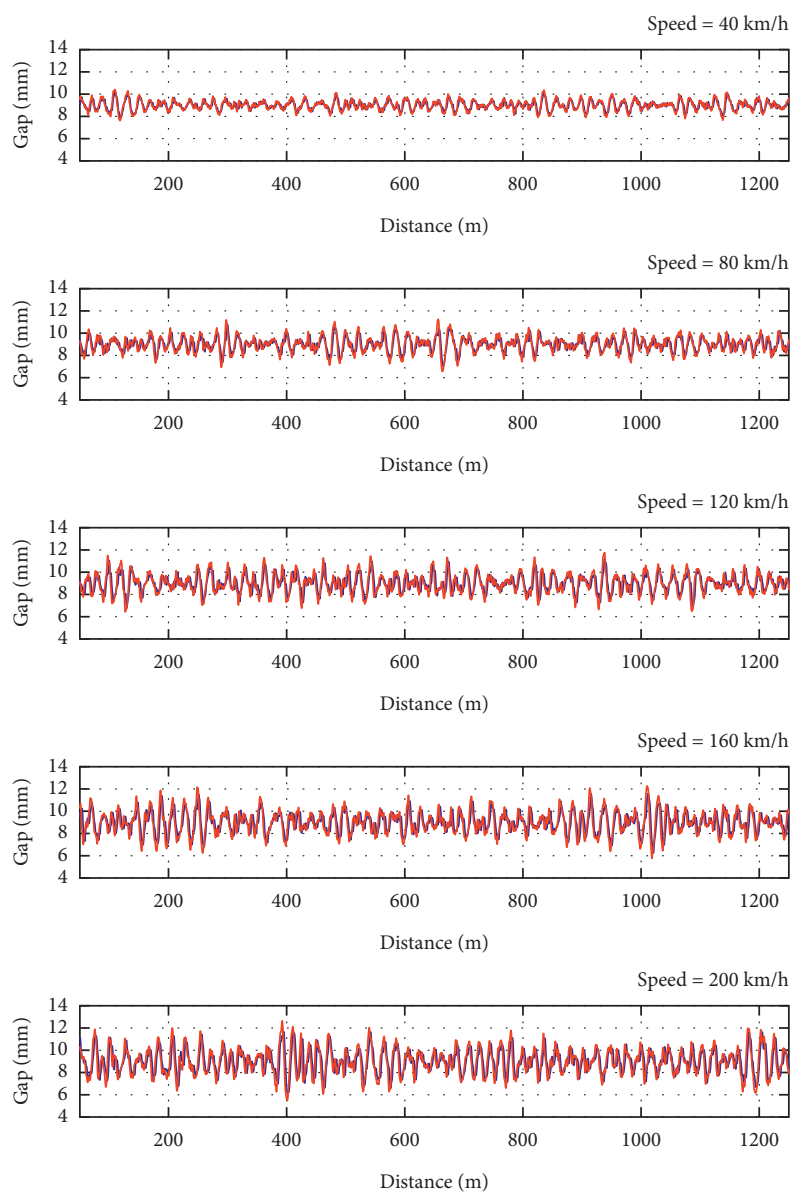

FIgURE 12: Gap fluctuation of no. 6 levitation module with different speed. 

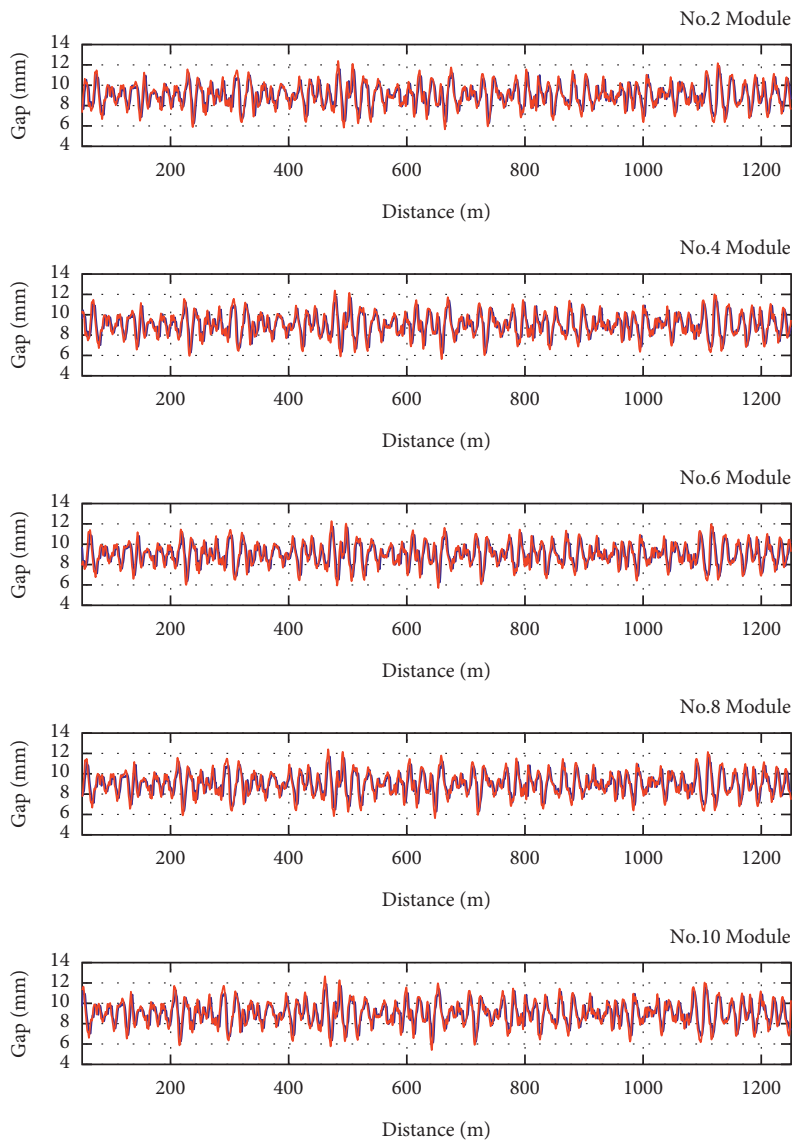

FIgURE 13: Gap fluctuation of one side levitation modules under $200 \mathrm{~km} / \mathrm{h}$.

Figure 14 shows the comparison of levitation energy consumption between pure electromagnetic levitation and hybrid levitation systems under different loads and static levitation conditions.

Compared with pure electromagnetic levitation, the power consumption during static suspension is greatly reduced. Affected by the characteristics of permanent magnets, the energy consumption of the hybrid suspension system decreases as the load increases. The energy consumption at $23 \mathrm{t}$ is reduced by $97 \%$. This is because the design of zero current hybrid electromagnet under light load conditions is generally adopted in the design. The energy consumption under $35 \mathrm{t}$ is reduced by $73 \%$. Within the full load and speed range, a large range of energy consumption reduction is achieved. For static conditions, the current of the electromagnet under different vehicle load conditions is greatly reduced. Furthermore, the power consumption of the suspension can be greatly reduced, so that the heating of the electromagnet can be greatly reduced. It is especially beneficial to reduce the heating when the vehicle is in static suspension after entering the station and solve the problem of high temperature of electromagnet.

Under dynamic operating conditions, the average power of the suspension system at different speeds and with different loads is shown in Figure 15, and the maximum power of the vehicle suspension system is shown in Figure 16.
In Figure 15, when the speed is increased, the greater the vehicle load, the greater the average power of the suspension. Under the same load condition, the speed change in the range of $40 \mathrm{~km} / \mathrm{h}-200 \mathrm{~km} / \mathrm{h}$ has little effect on the average power. Under the heavy load of $35 \mathrm{t}$, the average power in the range of $40 \mathrm{~km} / \mathrm{h}-200 \mathrm{~km} / \mathrm{h}$ is about $9.6 \mathrm{kw}$; under the same load condition, as the speed increases, the heat dissipation conditions of the electromagnet will be better, which is conducive to the stable and reliable operation of the suspension electromagnet. Therefore, in the thermal design of the electromagnet, the influence of the load should be mainly considered, and the speed has less influence on the internal heating of the electromagnet, and the air-cooled method and heat dissipation brought by the electromagnet should be put into consideration.

Figure 16 shows the max instantaneous power of the whole levitation system. It represents the max power that needed to be provided by the power supply system. The max power is $27.53 \mathrm{kw}$ under $200 \mathrm{~km} / \mathrm{h}$. Based on the simulation, we can decrease the levitation power supply system from $60 \mathrm{kw}$ used in the traditional pure electromagnet type maglev train to about $30 \mathrm{kw}$. It is a big improvement for the design of maglev train.

4.4. Analysis of Riding Comfort. The riding comfort is studied based on the vibration acceleration of the vehicle. As 

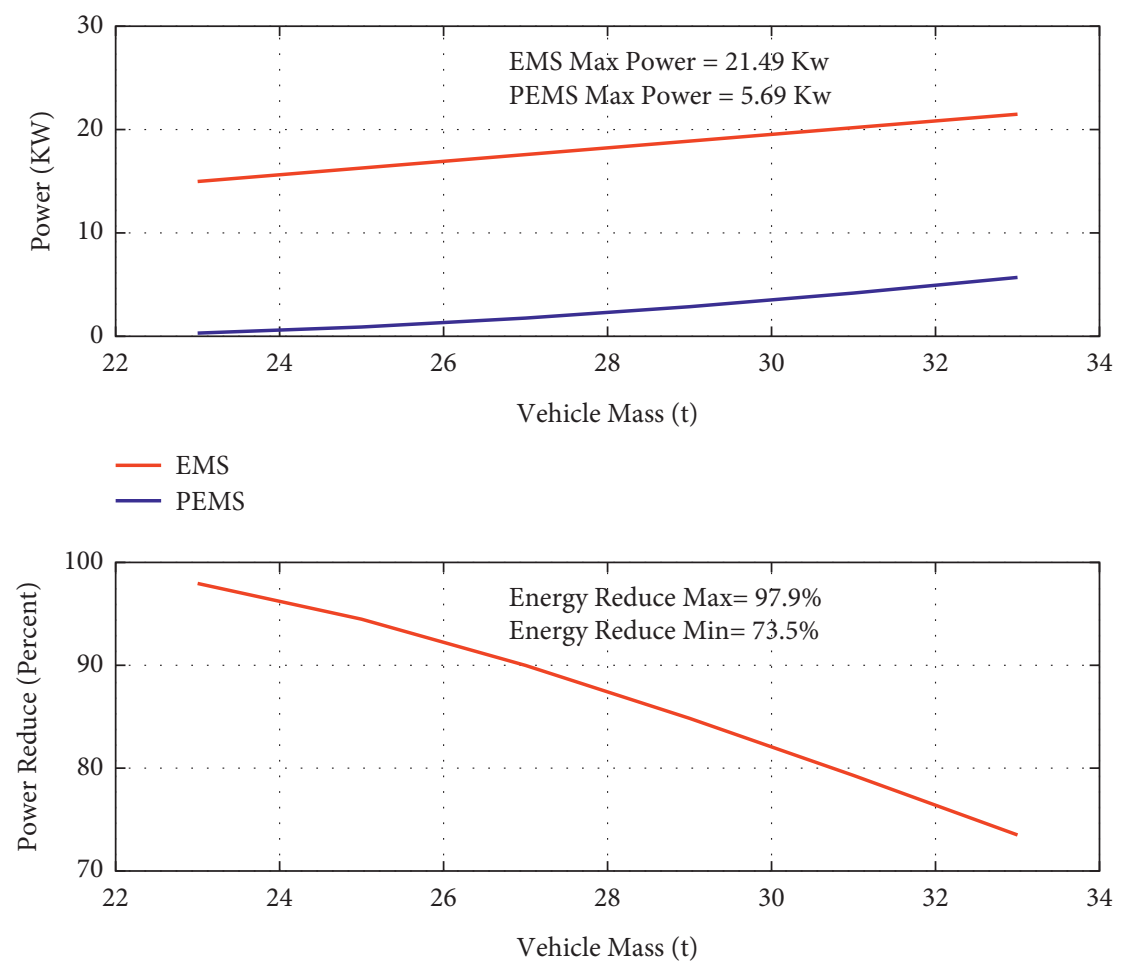

FiguRe 14: Energy consumption of static suspension under different loads.

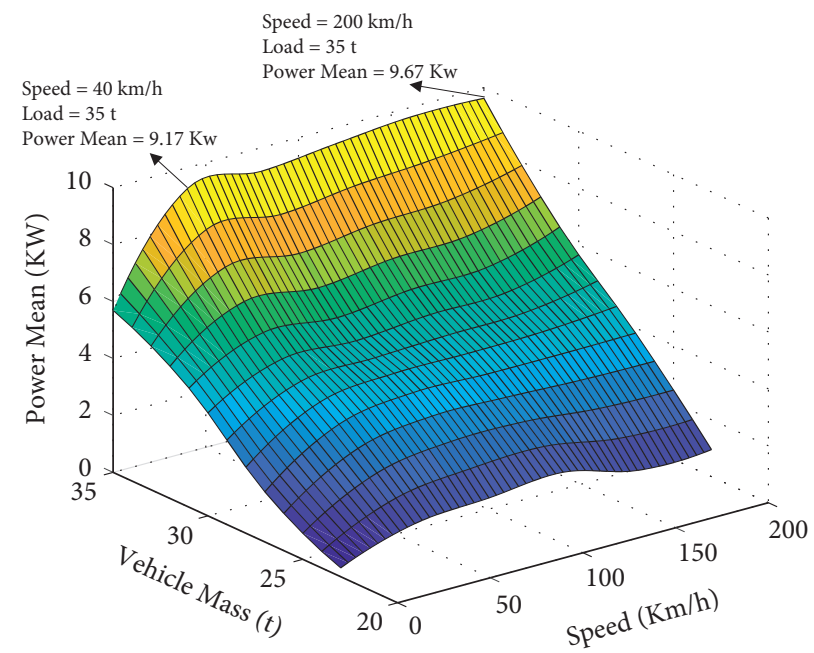

Figure 15: The average power of suspension system under different speeds and different loads.

shown in Figure 17, during the operation in different speed of the vehicle, the vertical acceleration of the vehicle body increases as the speed increases, but the vertical acceleration during the entire process does not exceed $0.04 \mathrm{~g}$. It is much smaller than the $0.13 \mathrm{~g}$ vertical vibration acceleration limit.

We adopt the Sperling stationarity index. The basic calculation formula is

$$
W=3.57\left(\sqrt[10]{\frac{a^{3} F(f)}{f}}\right) .
$$

Here, $W$ is the stability index, $a$ is the lateral or vertical vibration acceleration of the vehicle body, $f$ is the frequency of the vibration acceleration, and $F(f)$ is the frequency correction coefficient. The frequency correction factor is given in Table 2.

Since the vibration of the vehicle is random, its acceleration and frequency are constantly changing. The stability index of the entire data segment is calculated according to equation (22). $W_{i}$ is the main frequency stability index.

$$
W=\sqrt[10]{\left.W_{1}^{10}+W_{2}^{10}+\cdots+W_{n}^{10}\right)}
$$




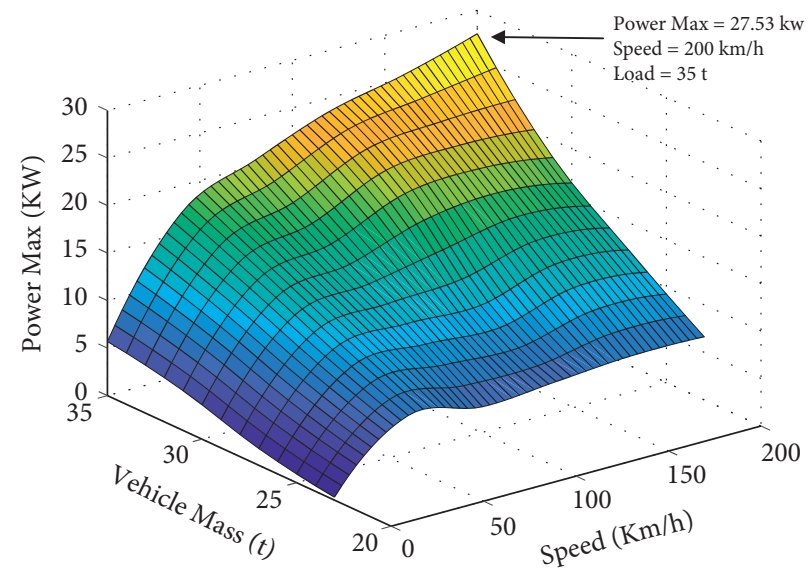

FIgURE 16: The maximum power of suspension system under different speeds and different loads.
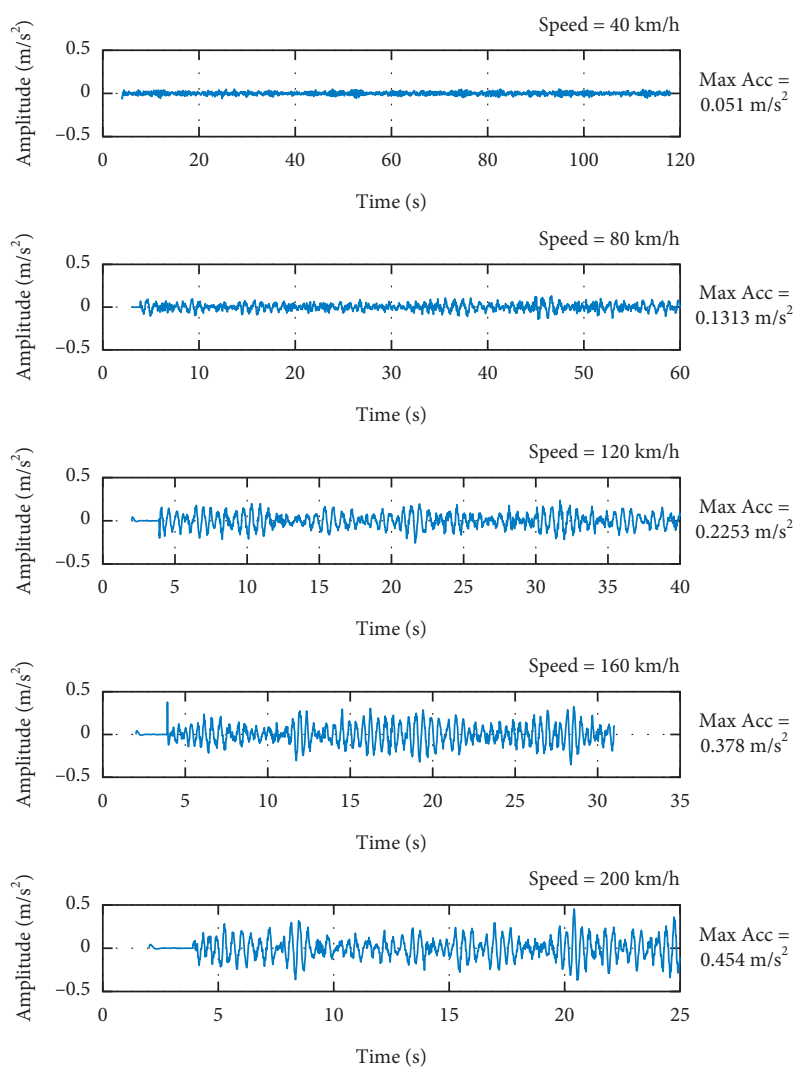

FIgURE 17: Vertical vibration of car body with $0-200 \mathrm{~km} / \mathrm{h}$ speed.

Through the analysis of the vertical acceleration, the range of the Sperling index at different speeds is shown in Figure 18.

The index increases with the increase of operating speed. It can be seen from the results that the comfort indexes are all in the excellent range $(<2.5)$. This means that, within $200 \mathrm{Km} / \mathrm{h}$, the vehicle has good riding comfort conditions under the excitation of track irregularities.

At the same time, it should be noted that, for the change curve of comfort, the Sperling index value at $140 \mathrm{~km} / \mathrm{h}$ speed reaches 2.32 , which is higher than 1.95 under $200 \mathrm{~km} / \mathrm{h}$ condition. The riding comfort of the
TABLE 2: Frequency correction factor.

\begin{tabular}{lcc}
\hline \multicolumn{3}{c}{ Vertical vibration } \\
\hline $0.5 \sim 5.9 \mathrm{~Hz}$ & $F(f)=0.325 f^{2}$ \\
$5.9 \sim 20 \mathrm{~Hz}$ & $F(f)=400 / f^{2}$ \\
$>20 \mathrm{~Hz}$ & $F(f)=1$ \\
\hline
\end{tabular}

vehicle near this speed is reduced. This is because, at the speed of $140 \mathrm{~km} / \mathrm{h}$, the frequency of stimulation caused by the speed and track irregularity is similar to the maglev train mode [23], thereby reducing the comfort of the car body. 


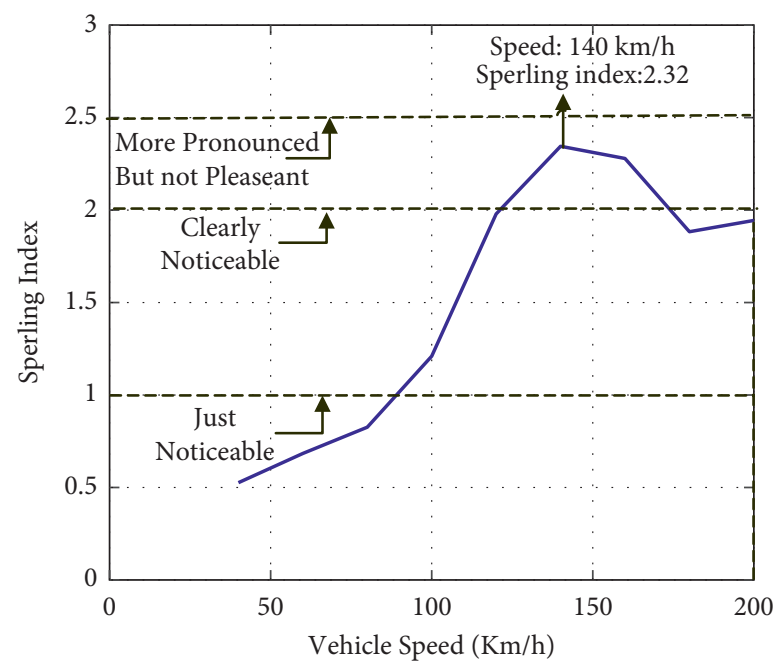

FIGURE 18: Sperling index under different speeds.

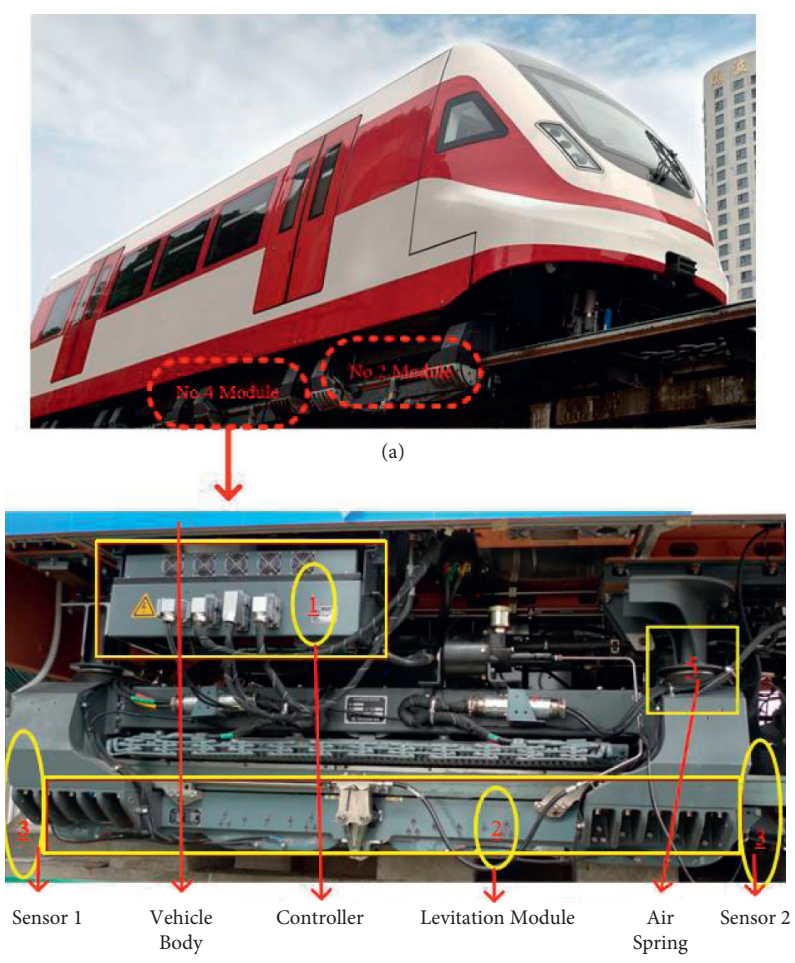

(b)

Figure 19: Medium-speed maglev train for experiment. (a) $160 \mathrm{~km} / \mathrm{h}$ type medium-speed maglev train and (b) Vehicle suspension structure.

\section{Experiments}

In order to verify the analytical calculation model and the simulation, this research took the medium-speed maglev train as the object and carried out the low-speed suspension performance and suspension energy consumption tests. Limited by the length of test line length, the highest speed in the experiment is $20 \mathrm{~km} / \mathrm{h}$.

The test vehicle is shown in Figure 19(a), and the levitation structure of the maglev train is shown in Figure 19(b). The levitation electromagnet of the maglev train uses a permanent magnet-electromagnetic hybrid levitation structure and two levitation sensors which are distributed at both ends of the suspended module. The levitation controller applies the PID control algorithm based on magnetic flux feedback, which can realize the stable levitation of the system. The suspension module is connected with the vehicle body through an air spring, which can achieve a better vibration isolation effect.

Limited by the length of the test line which is only $204 \mathrm{~m}$, the available running distance is only $150 \mathrm{~m}$. Therefore, the speed during the experiment is relatively low, and the 


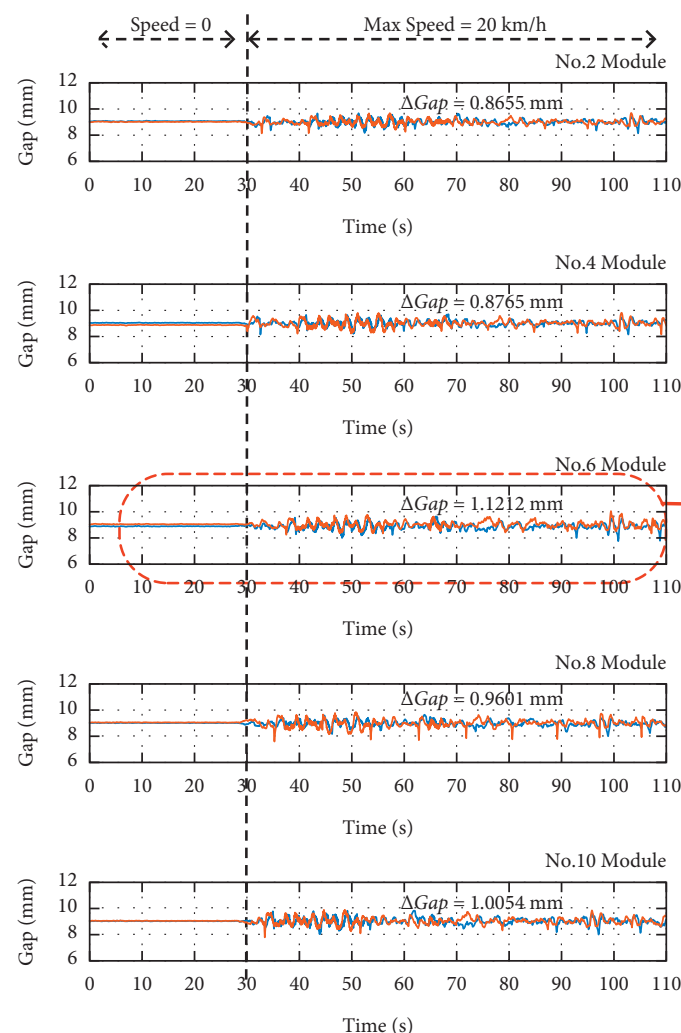

(a)

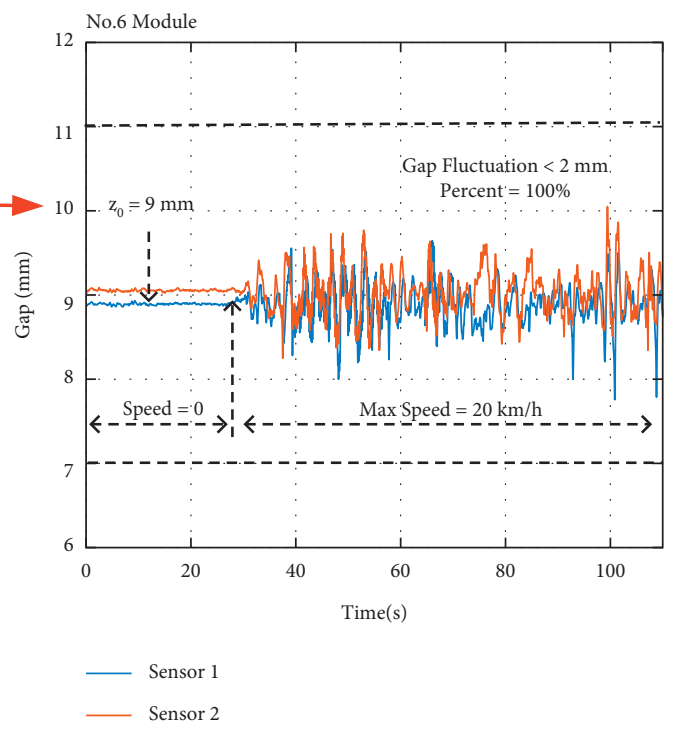

(b)

Figure 20: Levitation module gap. (a) Levitation module gap fluctuation (Nos. 2, 4, 6, 8, and 10). (b) No. 6 levitation module gap fluctuation.

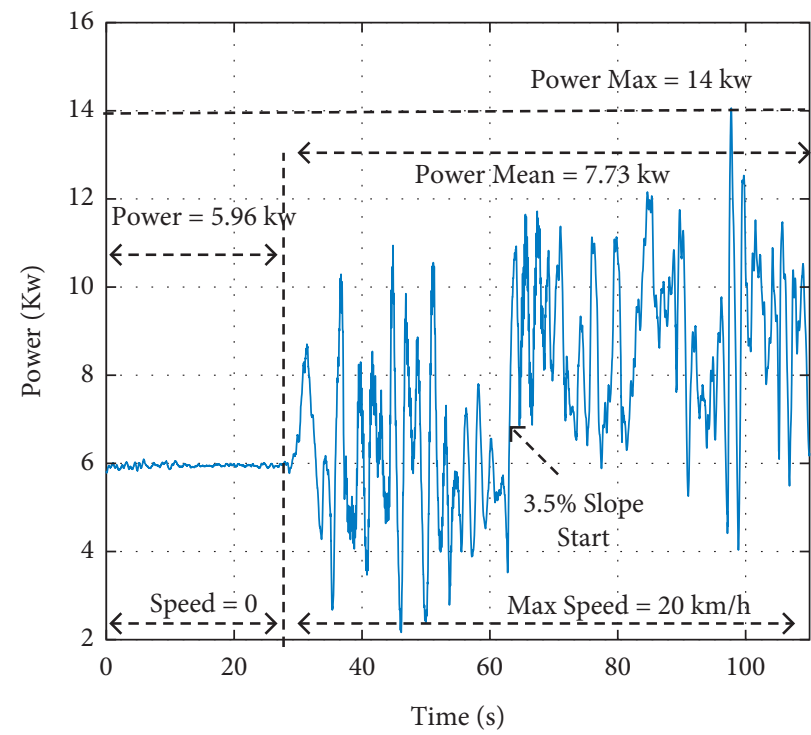

FIgURE 21: Suspension power with the max speed of $20 \mathrm{~km} / \mathrm{h}$

maximum speed is $20 \mathrm{~km} / \mathrm{h}$. The vehicle is designed for working under static and dynamic conditions with heavy load AW3 (35 t). It is under static suspension from 0 to $30 \mathrm{~s}$. At $30 \mathrm{~s}$, it starts to accelerate. The total dynamic running time is $80 \mathrm{~s}$, and the maximum speed is $20 \mathrm{~km} / \mathrm{h}$. Figure 20 is the gap of levitation module during the whole test time.

Among the five suspension modules on one side of the train, the steady-state suspension gap is set to $9 \mathrm{~mm}$, and the suspension gap fluctuations of all suspension modules are less than $\pm 1.5 \mathrm{~mm}$. This shows that the vehicle suspension system has good control performance and output characteristics.

Figure 21 shows the controller power output during the operation of the vehicle. Under static suspension, the vehicle suspension power is $5.96 \mathrm{KW}$, which is slightly larger than the $5.69 \mathrm{kw}$ calculated by the simulation, and the error is less 
than $5 \%$ (mainly because of the controller chopper loss). Under static conditions, the simulation and measured results of the system are similar. During the dynamic operation of the system, the maximum instantaneous suspension power of the whole vehicle is $14 \mathrm{kw}$, and the average power is $7.73 \mathrm{kw}$. In the dynamic operation process, due to the need of levitation control, the internal current of the levitation electromagnet fluctuates, which greatly increases the levitation power during the entire operation. The experimental results are consistent with the simulation results, verifying the correctness of the simulation results under low-speed conditions.

\section{Conclusions}

In this study, an analytical calculation model of the mediumspeed maglev train was established, and a PID levitation control system based on magnetic flux density feedback was designed. Taking the actual track irregularity as input, numerical simulation was applied to study the performance of the suspension system, suspension energy consumption, and riding comfort under different loads and at different speeds. Experiment results have shown that the calculation model and the numerical simulation are effective.

In this paper, a suitable levitation control strategy has firstly been proposed and verified for medium-speed $(0-200 \mathrm{~km} / \mathrm{h})$ maglev train. It is shown that the fluctuation range of the train suspension gap is less than $\pm 4 \mathrm{~mm}$. The suspension power consumption of the whole vehicle with different loads and at different speeds has firstly been studied. The results show that the instantaneous levitation power of the vehicle is less than $30 \mathrm{kw}$, which is far lower than the $60 \mathrm{kw}$ required before. The riding comfort in the range of $0-200 \mathrm{~km} / \mathrm{h}$ has firstly been considered. It is demonstrated that the Sperling index is less than 2.5 in the speed range of $0-200 \mathrm{~km} / \mathrm{h}$.

\section{Data Availability}

The data used to support the findings of this study are available from the corresponding author upon request.

\section{Conflicts of Interest}

The authors declare that they have no conflicts of interest.

\section{Acknowledgments}

This work was supported by the National Key R\&D Program of China (Grant no. 2016YFB1200601).

\section{References}

[1] J. Kluhspies, "Disruptive technologies and their long-term perspectives-the case of maglev transportation systems," in Proceedings of the Maglev 2016, Berlin, Germany, September 2016.

[2] G. Lin and X. Sheng, "Application and further development of maglev transportation in China," Transportation Systems and Technology, vol. 4, no. 3, pp. 36-43, 2018.
[3] N. Motonori, "Ten years commercial operation of LINIMO in aichi pref Japan," in Proceedings of the Maglev 2016, Berlin, Germany, September 2016.

[4] H. Han, "Development of a high-speed maglev vehicle in Korea," in Proceedings of the Maglev 2016, Berlin, Germany, September 2016.

[5] S. Richard and F. Costa, "The experimental line of the maglevcobra project for urban transportation," in Proceedings of the Maglev 2016, Berlin, Germany, September 2016.

[6] B. C. Shin and D. Y. Park, "Incheon international airport maglev line," in Proceedings of the Maglev 2016, Berlin, Germany, September 2016.

[7] MagneMotion Inc, The M3 Urban Transportation System, Magnemotion Document UM-1, Devens, MA, USA, 2003.

[8] R. Thornton, T. Clark, and B. Perreault, "An M3 maglev system for old dominion university," in Proceedings of the Maglev'2008, San Diego, CA, USA, 2008.

[9] P. Yu, S. Chen, M. Gao, Q. Yang, L. Wang, and J. Li, "Optimal design of end suspension electromagnet for medium speed maglev train," in Proceedings of the 2020 39th Chinese Control Conference (CCC), Henyang, China, July 2020.

[10] J.-h. Li, J. Li, P.-c. Yu, and L.-c. Wang, "Adaptive backstepping control for levitation system with load uncertainties and external disturbances," Journal of Central South University, vol. 21, no. 12, pp. 4478-4488, 2014.

[11] Y. Li and W. Chang, "Cascade control of an EMS maglev vehicle's levitation control system," Acta Automatica Sinica, vol. 25, no. 2, pp. 247-251, 1999, in Chinese.

[12] P. K. Sinha and A. N. Pechev, "Model reference adaptive control of a maglev system with stable maximum descent criterion," Automatica, vol. 35, no. 8, pp. 1457-1465, 1999.

[13] H. W. Cho, H. S. Han, J. M. Lee, B. S. Kim, and S. Y. Sung, "Design considerations of EM-PM hybrid levitation and propulsion device for magnetically levitated vehicle," IEEE Transactions on Magnetics, vol. 45, no. 10, pp. 4632-4635, 2009.

[14] W. Zhang, J. Li, K. Zhang, and P. Cui, "Design of magnetic flux feedback controller in hybrid suspension system," Mathematical Problems in Engineering, vol. 2013, Article ID 712764, 2013.

[15] C. F. Zhao and W. M. Zhai, "Maglev vehicle/guideway vertical random response and ride quality," Vehicle System Dynamics, vol. 38, no. 3, pp. 185-210, 2002.

[16] J. Shi, Q. Wei, and Y. Zhao, "Analysis of dynamic response of the high-speed EMS maglev vehicle/guideway coupling system with random irregularity," Vehicle System Dynamics, vol. 45, no. 12, pp. 1077-1095, 2007.

[17] G. He, J. Li, and P. Cui, "Decoupling control design for the module suspension control system in maglev train," Mathematical Problems in Engineering, vol. 2015, no. 2, pp. 1-13, Article ID 865650, 2015.

[18] R. M. Goodall, "On the robustness of flux feedback control for electromagnetic maglev controllers," in Proceedings of the Maglev 2000, pp. 197-202, Copacabana Beach, Rio de Janeiro, Brazil, June 2000.

[19] Z.-J. Yang, K. Kunitoshi, S. Kanae, and K. Wada, "Adaptive robust output-feedback control of a magnetic levitation system by K-Filter approach," IEEE Transactions on Industrial Electronics, vol. 55, no. 1, pp. 390-399, 2008.

[20] P. K. Sinha, Electromagnetic Suspension: Dynamics and Control, no. 30, pp. 101-106, IEE Control Engineering Series, Peter Peregrinus Ltd., London, UK, 1986.

[21] L. Wang, P. Yu, J. Li, and P. Cui, "A practical magnetic flux density observer for hybrid suspension system of maglev 
train," in Proceedings of the 36th Chinese Control Conference, Dalian, China, July 2017.

[22] G. Zhang, J. Li, and Z. Yang, "Estimation of power spectrum density track irregularities of low-speed maglev railway lines," Journal of the China Railway Society, vol. 33, pp. 73-78, 2011.

[23] Y. Li, D. Zhou, and J. Li, "Vertical dynamic response prediction of the electromagnetic levitation systems," Applied Science, vol. 10, Article ID 2580, 2020. 